PROCEEDINGS OF THE AMERICAN MATHEMATICAL SOCIETY

Volume 124, Number 6, June 1996

\title{
ALMOST DISJOINT PERMUTATION GROUPS
}

\author{
FRED GALVIN
}

(Communicated by Andreas R. Blass)

\begin{abstract}
A permutation group $G$ on a set $E$ of (infinite) cardinality $\kappa$ is almost disjoint if no element of $G$ except the identity has $\kappa$ fixed points, i.e., if $G$ is an almost disjoint family of subsets of $E \times E$. We show how almost disjoint permutation groups can be constructed from almost disjoint families of sets.
\end{abstract}

Permutations are composed from left to right; $\operatorname{Sym}(E)$ is the group of all permutations of $E$; the set of fixed points of a permutation $\pi$ is denoted by $\operatorname{fix}(\pi)$. The letters $\delta, \kappa, \lambda$ will denote infinite cardinals.

A family $\mathcal{S}$ of sets is $\delta$-almost disjoint if $|X \cap Y|<\delta$ whenever $X, Y \in \mathcal{S}$ and $X \neq Y$. A family of $\kappa$-element sets is almost disjoint if it is $\kappa$-almost disjoint. A permutation group $G$ on an infinite set $E$ is $\delta$-almost disjoint if $|\operatorname{fix}(\pi)|<\delta$ for every $\pi \in G \backslash\{1\}$; this amounts to saying that, if we regard the permutations as subsets of $E \times E$, then $G$ is a $\delta$-almost disjoint family of sets. The group $G$ is almost disjoint if it is $\kappa$-almost disjoint for $\kappa=|E|$.

In this note, we show that some questions about the existence of almost disjoint permutation groups reduce to questions about almost disjoint families of sets, which have been thoroughly investigated by set theorists: see Baumgartner [1] or Williams [4] for a survey of results in this area.

Theorem 1. For any uncountable cardinals $\delta \leq \kappa \leq \lambda$, the following statements are equivalent:

(i) there is a $\delta$-almost disjoint subgroup $G$ of $\operatorname{Sym}(\kappa)$ with $|G|=\lambda$;

(ii) there is a $\delta$-almost disjoint family $\mathcal{S}$ of $\kappa$-element subsets of $\kappa$ with $|\mathcal{S}|=\lambda$.

Proof. Only the implication (ii) $\Rightarrow$ (i) requires proof. Let $I$ and $T$ be sets, $|I|=\lambda$, $|T|=\kappa$. Choose sets $S_{i} \subseteq T(i \in I)$ so that $\left|S_{i}\right|=\kappa$ and $\left|S_{i} \cap S_{j}\right|<\delta$ for $i \neq j$. For each $i \in I$, choose an involution $\theta_{i} \in \operatorname{Sym}(T)$ so that $S_{i} \theta_{i}=T \backslash S_{i}$, and then define an involution $\pi_{i} \in \operatorname{Sym}(T \times \mathbb{Z})$ by setting

$$
(t, n) \pi_{i}= \begin{cases}\left(t \theta_{i}, n+1\right) & \text { if } t \in S_{i}, \\ \left(t \theta_{i}, n-1\right) & \text { if } t \notin S_{i} .\end{cases}
$$

Let $G$ be the subgroup of $\operatorname{Sym}(T \times \mathbb{Z})$ generated by $\left\{\pi_{i}: i \in I\right\}$. As $|T \times \mathbb{Z}|=\kappa$ and $|G|=\lambda$, we only have to show that $G$ is $\delta$-almost disjoint. It will suffice to prove the following.

Received by the editors December 20, 1994.

1991 Mathematics Subject Classification. Primary 20B07.

(C)1996 American Mathematical Society 
Claim. Let $m \geq 1$. If $i_{1}, \ldots, i_{m} \in I$ and $i_{j} \neq i_{j+1}$ for $1 \leq j<m$, then $\left|\operatorname{fix}\left(\pi_{i_{1}} \pi_{i_{2}} \cdots \pi_{i_{m}}\right)\right|<\delta$.

We prove the claim by induction on $m$. It is clear for $m=1$, since $\pi_{i}$ has no fixed points. Suppose $m>1$. We may assume that $i_{m} \neq i_{1}$, as otherwise we have $m>2$ and the induction hypothesis applies to the conjugate permutation $\pi_{i_{2}} \cdots \pi_{i_{m-1}}$. Let $i_{m+1}=i_{1}$; then $i_{j} \neq i_{j+1}$ for $1 \leq j \leq m$. Let

$$
R=\bigcup_{j=1}^{m}\left(S_{i_{j}} \cap S_{i_{j+1}}\right) \theta_{i_{j}} \cdots \theta_{i_{2}} \theta_{i_{1}}
$$

We will show that $\operatorname{fix}\left(\pi_{i_{1}} \pi_{i_{2}} \cdots \pi_{i_{m}}\right) \subseteq R \times \mathbb{Z}$; this will finish the proof, since $|R \times \mathbb{Z}|<\delta$.

Suppose $\left(t_{0}, n_{0}\right) \in \operatorname{fix}\left(\pi_{i_{1}} \pi_{i_{2}} \cdots \pi_{i_{m}}\right)$; we have to show that $t_{0} \in R$. For $1 \leq j \leq$ $m+1$, let $\left(t_{j}, n_{j}\right)=\left(t_{0}, n_{0}\right) \pi_{i_{1}} \pi_{i_{2}} \cdots \pi_{i_{j}}=\left(t_{j-1}, n_{j-1}\right) \pi_{i_{j}}$. Then $n_{m}=n_{0}, n_{m+1}=$ $n_{1}$, and for $1 \leq j \leq m+1$ we have

$$
n_{j}-n_{j-1}= \begin{cases}+1 & \text { if } t_{j-1} \in S_{i_{j}}, \\ -1 & \text { if } t_{j-1} \notin S_{i_{j}} .\end{cases}
$$

Pick $j \in\{1,2, \ldots, m\}$ with $n_{j}=\min \left\{n_{1}, n_{2}, \ldots, n_{m}\right\}$. Then $n_{j}<n_{j+1}$, whence $t_{j} \in S_{i_{j+1}}$; and $n_{j}<n_{j-1}$, whence $t_{j-1} \notin S_{i_{j}}$, whence $t_{j}=t_{j-1} \theta_{i_{j}} \in S_{i_{j}}$. Thus we have $t_{0} \theta_{i_{1}} \theta_{i_{2}} \cdots \theta_{i_{j}}=t_{j} \in S_{i_{j}} \cap S_{i_{j+1}}$, and so $t_{0} \in\left(S_{i_{j}} \cap S_{i_{j+1}}\right) \theta_{i_{j}} \cdots \theta_{i_{2}} \theta_{i_{1}} \subseteq R$.

The following corollary answers a question of mine [2, Question 1.5], which was motivated by some off-hand remarks of P. M. Neumann. The corollary follows from Theorem 1 together with Sierpiński's result [3, p. 241] that, if $2^{\omega}=\omega_{1}$, then there are $2^{\omega_{1}}$ almost disjoint uncountable subsets of $\omega_{1}$. By a consistency result of Baumgartner [1, Theorem 5.6], the continuum hypothesis is indispensible here.

Corollary. If $2^{\omega}=\omega_{1}$, then $\operatorname{Sym}\left(\omega_{1}\right)$ has an almost disjoint subgroup of cardinality $2^{\omega_{1}}$.

Theorem 2. For any infinite cardinals $\kappa$ and $\lambda$, the following statements are equivalent:

(i) there is an almost disjoint subgroup $G$ of $\operatorname{Sym}(\kappa)$ with $|G|=\lambda$;

(ii) there is an almost disjoint family $\mathcal{S}$ of $\kappa$-element subsets of $\kappa$ with $|\mathcal{S}|=\lambda$.

Proof. In view of Theorem 1, all we have left to prove is that $\operatorname{Sym}(\omega)$ has an almost disjoint subgroup of cardinality $2^{\omega}$. For each $n \in \omega$, let $\left(B_{n},+\right)$ be the free Boolean group (any nontrivial locally finite variety of groups will do) generated by an $(n+1)$-element set $A_{n}$. Let $E$ be the disjoint union of the sets $B_{n}$. As shown by Sierpiński [3, p. 242], we can define a subset $F$ of the Cartesian product $\prod_{n<\omega} A_{n}$, with $|F|=2^{\omega}$, so that $|f \cap g|<\omega$ whenever $f, g \in F$ and $f \neq g$. For each $f \in F$, define $\pi_{f} \in \operatorname{Sym}(E)$ so that $x \pi_{f}=x+f(n)$ for $x \in B_{n}$. Let $G$ be the subgroup of $\operatorname{Sym}(E)$ generated by $\left\{\pi_{f}: f \in F\right\}$; it is easy to verify that $G$ is almost disjoint.

\section{ACKNowledGMents}

I thank Peter M. Neumann for his helpful advice. This project was sponsored by the National Security Agency under Grant Number MDA904-92-H3037. The United States Government is authorized to reproduce and distribute reprints. 


\section{REFERENCES}

1. James E. Baumgartner, Almost-disjoint sets, the dense set problem and the partition calculus, Ann. Math. Logic 9 (1976), 401-439. MR 53:5299

2. Fred Galvin, Generating countable sets of permutations, J. London Math. Soc. (2) 51 (1995), 230-242. CMP 95:10

3. W. Sierpiński, Sur une décomposition d'ensembles, Monatsh. Math. Phys. 35 (1928), 239-242.

4. Neil H. Williams, Combinatorial set theory, North-Holland, Amsterdam, 1977.

Department of Mathematics, University of Kansas, Lawrence, Kansas 66045-2142

E-mail address: galvin@math.ukans.edu 